\title{
The Eurescom Project HINE (Heterogeneous In-House Networking Environment): A Cooperation between Telecom Operators for Advanced Home Networking
}

\author{
$\underline{\text { Paolo Pastorino }}^{1}$, Steve Brown ${ }^{2}$, Pierre-Yves Danet ${ }^{3}$, Gabriele Goldacker ${ }^{4}$, José \\ Gonzales Torres ${ }^{5}$, Torvald Konstali ${ }^{6}$, Frederic Phytoud $^{7}$ \\ ${ }^{1}$ CSELT, Via Reiss Romoli 274, 10148 Torino - ITALY, \\ ${ }^{1}$ paolo.pastorino@cselt.it \\ ${ }^{2}$ British Telecom, UK \\ ${ }^{3}$ France Télécom R\&D, France \\ ${ }^{4}$ GMD-Fokus, Germany \\ ${ }^{5}$ Telefonica I/D, Spain \\ ${ }^{6}$ Telenor, Norway \\ ${ }^{7}$ Swisscom, Switzerland
}

\begin{abstract}
An overview of an integrated of home networking environment from a Telecom Operator and Service Provider's point of view is presented. This is a result of a cooperative European project (Eurescom P915 HINE) integrating heterogeneous home networks. It gives an insight of the basic requirement of an integrated home network that can enable the delivery of advanced telecommunication services, furthermore the results of a European distributed demonstrator are reported.
\end{abstract}

\section{Introduction and Basis}

The scenario of people seamlessly being able to control all elements of their heterogeneous home environment remotely via the Internet - like their video, heating, delivery of shopping and so on, is fast becoming reality. Over the past year, seven European Telecom Operators have been actively collaborating with each other and component vendors in the Eurescom project HINE (Heterogeneous In-house Networking Environment) [1] to fully understand the potential for the home networking environment and whether there is a market out there.

The current target environments are mainly the in-house, business and home areas with wired and wireless systems. In addition to classical Customer Premises Equipment such as telephone, fax, PC and so on, there are many largely standalone microelectronics-controlled equipment (stereo / video products, home theatre, white goods, light control, burglar alarm, person identification / location systems etc.) that are applied in households. 


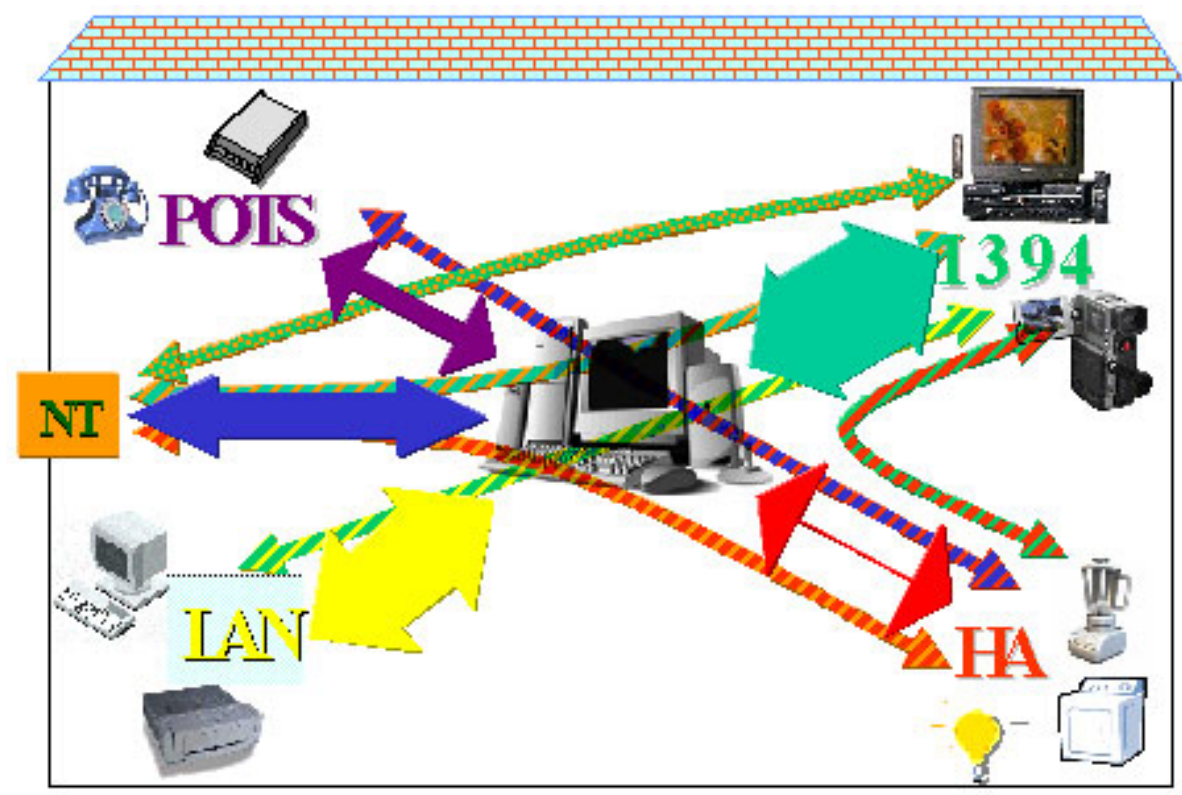

Fig. 1. The Heterogeneous home network

Integrating these devices in an homogeneous and interworking communication environment using mainly existing or easy-to-install networks (e.g. power-line, wireless systems), will allow major enhancements to existing and the creation of completely new applications and services.

The project has focused on analyzing the key technologies for the realization of heterogeneous in-house networking systems for the home and office environment, providing a platform for communication, automation, control and facility management applications and services and operation of an Internet integrated HINE test-bed for commercial and pre-commercial components, applications and services.

\section{The Theoretical Work}

Beginning with existing or "easy to install" wired or wireless physical media and transmission systems, there is already a huge variety of possible means to access those devices. Some standards even allow the simultaneous use of the physical media for different transmission technologies, while other standards require special topologies, which might no be well suited for some countries. On top of the transmission technologies, it will be necessary to provide interoperability between devices in the same system and even across system boarders. Due to the focus on private customers and consumers, plug and play capabilities are a must as well. In addition, the Telecom Operators will have to provide complete solutions as a service to their customers. 
In-house technologies have been compared and conclusions so far suggest that the commercial reality of the HINE environment is likely to comprise a mix of both wired and wireless networking. The constraints, approaches and impacts for the gateway and application programming interfaces to hook the in-house network into the access network have been evaluated, along with the influences of home networks on access networks. Furthermore scenarios have to be developed to allow customers to buy complete solutions in a one-stop-shopping manner from a Telecom Operator.

Therefore one goal of the EURESCOM Project HINE has been to analyse the currently available solutions and future trends in the area of home communication and automation with an explicit focus on the possibility to attach theses systems to the access networks provided by the Telecom Operators [2-4]. The theoretical work, that has been carried out by the Project members provided an overview over possible solutions, which have taken into account the above mentioned criteria. In addition this work has been the basis for the development and implementation of the HINE demonstrator.

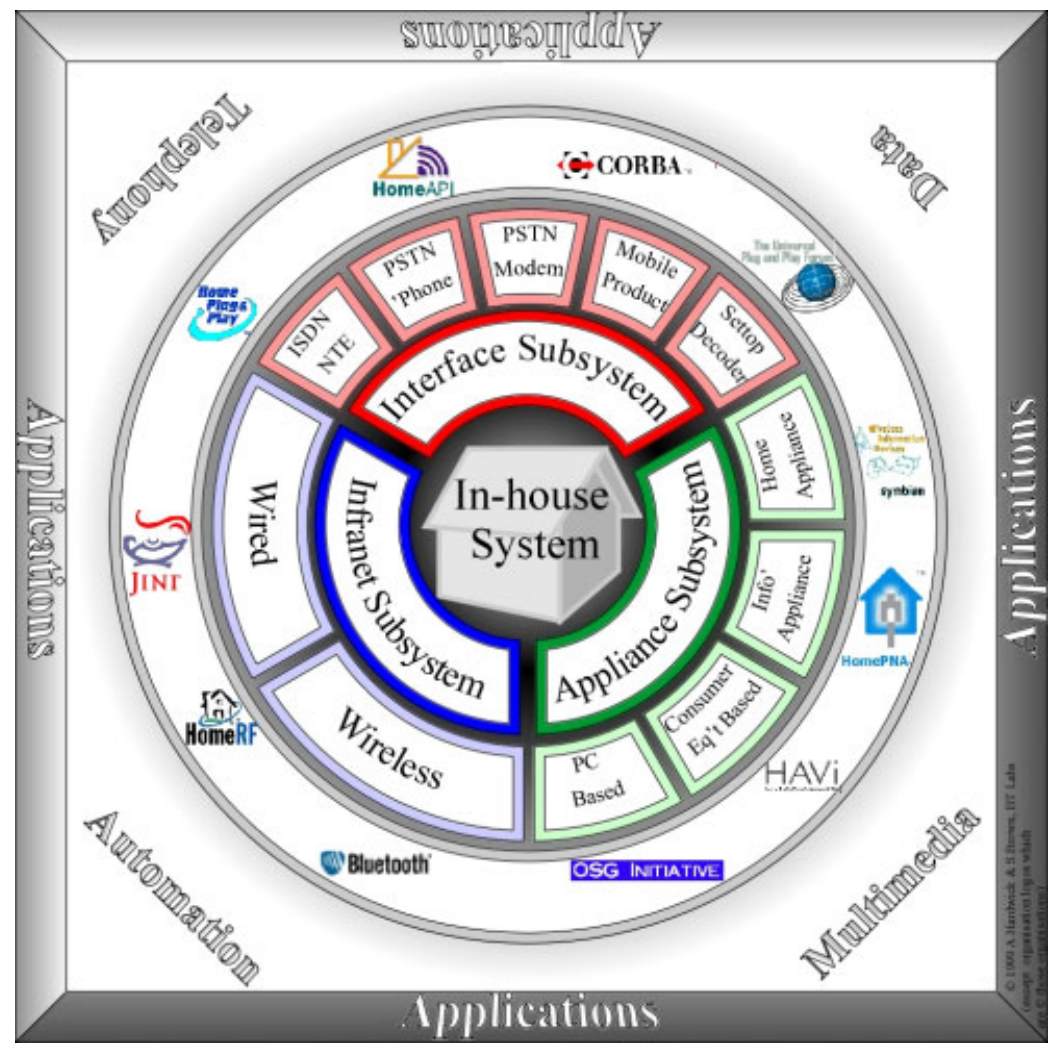

Fig. 2. The In-house system structure 


\section{Residential Gateway \& APIs}

The Residential Gateway (RG) is the key component in the home for connecting the various internal networks to different external access networks. However, it also serves as a platform for deploying different kinds of services to the end user.

The requirements necessary to put on the Gateway are very hard to fulfill - the Gateway should be cheap, have a nice looking, make no noise, be easy to use, easy to manage, reliable, secure, and should be able to perform several challenging tasks simultaneously.

To achieve this, it is essential that the several actors to some extent follow the same approaches. This is not the case today, as there exists several initiatives that work in parallel on different solutions on the several aspects relating to this.

To make a RG within acceptable cost limits, the number of simultaneously available network interfaces has to be reduced to the minimum that is required to provide an overall set of services. To provide flexibility it is essential to use a standard internal interface for network interface cards, and hot plugging is a desire. The RG might be single physical unit, but might also be a distributed system containing several more or less interconnected units.

Alarm services are potential services for this system, but introducing such services puts constraints on the availability and security features of the system. The RG has to be placed in a restricted area, and has to have a back up power supply.

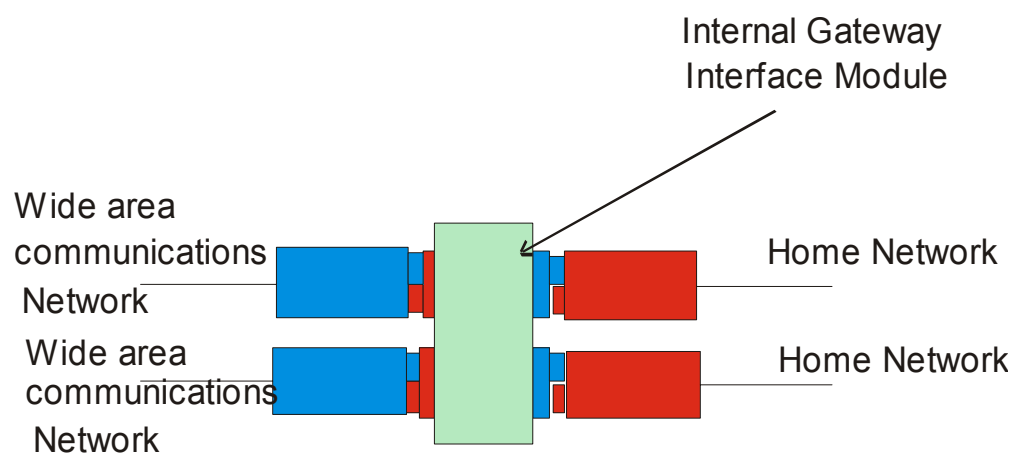

Fig. 3. The centralized Residential Gateway 


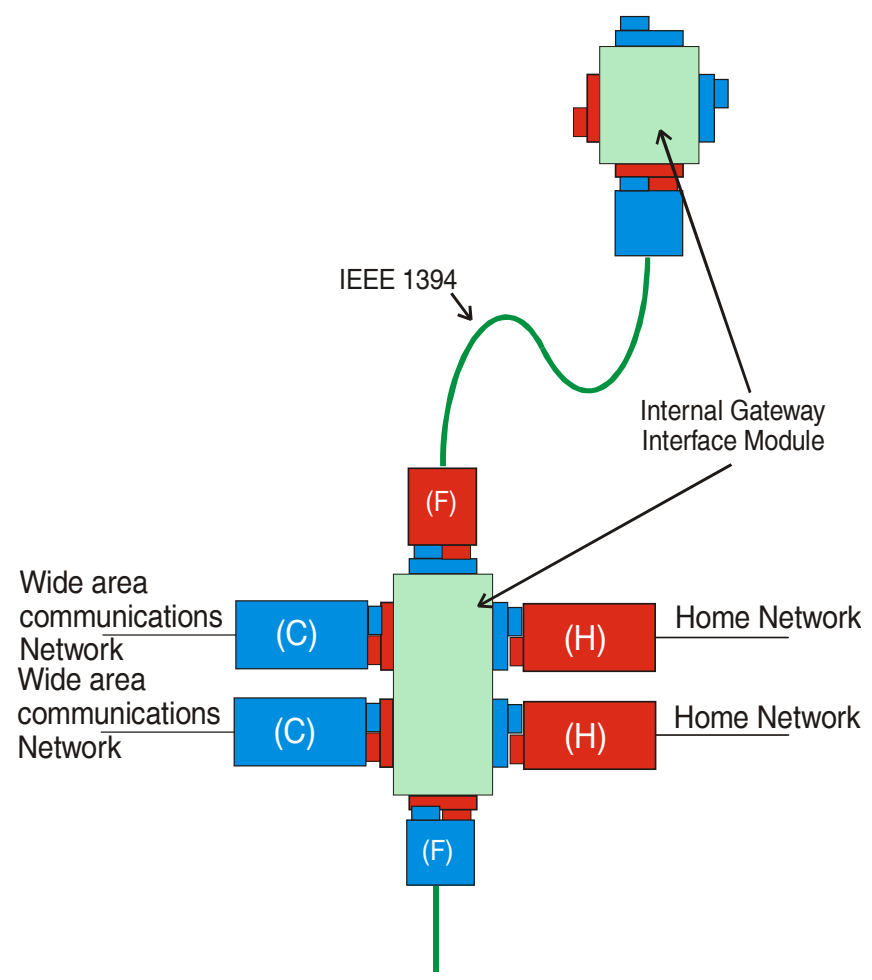

Fig. 4. The distributed Residential Gateway

Today existing home applications are based on a number of different APIs that are often proprietary, incompatible and normally just address subsets of the devices that exists in a home. This leads to a demand for a general API giving standardized access to all home devices independent of the network type used. Such an API should have functionality for invoking methods, provide status, do management and acquire specific attributes for devices. In addition persistence, security, automation and resource handling has to be taken into account. There are several initiatives to define the necessary API. Among them we have the Home API (now merged with Universal Plug and Play) and the OSGi (Open Service Gateway Initiative) initiatives. Both of them are in an early stage of development and it is too soon to judge the importance of these efforts. Nonetheless OSGi has succeeded in releasing its first specification and many manufacturers and providers are starting the first implementation of OSGi compliant platforms.

Another very important aspect of the home system is the "the plug and play" capabilities. Also here there are several initiatives; Microsoft leads the work on "Universal Plug and Play" and Sun promotes Jini. "Universal Plug and Play" is still in an early stage of development and not much information is available (a Device Architecture specification has just been released in June 2000). Jini is a Java based resource discovers system that may be a component in other systems as HAVi (an 
initiative coming from the consumer electronics market) and OSGi. It is not clear today if any solution will win over the others, and in that case, which it will be. Because of this several equipment manufacturers works with several solutions in parallel. For example, companies like Echelon and Axis seems to promote both Jini and Universal Plug and Play.

The overall situation in this area is rather chaotic, and there is really a need to establish some overall guidelines to get a more smooth evolution. Telecom Operators and Service Providers can be puzzled when it comes the time for choosing the right platform.

\section{The Practical Work}

Besides the important theoretical work, the main goal of the Project has been to realise a distributed HINE demonstrator, interconnected via the Internet, broadband access networks and ISDN or POTS. All HINE Project partners have set up local scenarios to demonstrate the functionality and usability of global accesses to a variety of home communication systems. Due to the large number of standards, transmission technologies and physical media, the resulting demonstrator is thus completely heterogeneous and it gives useful and necessary experiences to all partners involved for future product and service developments. The distributed HINE demonstrator comprises a mix of online applications and hardware demonstrators. Due to the distributed physical approach of the demonstrator, the Internet has been used to interconnect the different demonstrator sites. This allowed creating a central demonstrator site for the access through the WWW.

\section{The Distributed Demonstrator}

The development and operation of a distributed demonstrator was a central goal of HINE. The expectation that realistic experiences with home networking technologies can best be made in this way became absolutely true.

\section{Scope of demonstrator evaluation}

After having separately tested and evaluated several technologies relevant or promising for the home networking area, the scope of the demonstrator evaluation was focused on the capabilities of the overall systems developed.

The major issues identified are: man-machine interface, reliability, security, API implementations and access network basic requirements. Besides these areas, several other conclusions of broad relevance have been drawn. The major observations are presented in the following sections. 


\section{The distributed demonstrator concept}

Each HINE partner has set up a self-contained demonstrator part realizing several scenarios on a common technical basis. All demonstrator parts are accessible via a common World Wide Web access point (a web portal) that is partly limited by a password access control (some of the HINE Project results a confidential and reserved to the shareholders only). Additionally, dedicated access points for voice telephony, SMS and WAP are provided.

In a real life environment, a portal (a web portal is the more straightforward way of doing it) is a comfortable way to realize secure remote access without explicit authentication per location. It can be for example implemented at the home site of a given user or be provided by a (security) service provider (the basic assumption for a web portal is that the user needs to have for most of the scenarios an always-on connection).

The distributed demonstrator made it possible to realize similar scenarios using different technologies and thus to directly compare their suitability for a certain purpose.

\section{Demonstrator scenarios}

A broad selection of scenarios covering all areas of home networking - home automation, multimedia /entertainment and communication - has been realized. Here in the following a short description is included. We have not included on purpose broadband video services (e.g. digital video broadcasting, Video on Demand) because they are not aligned with the main focus of the project (heterogeneous networking).

\section{Home automation}

It includes in-home control, external control by the occupants, automatic provision of information to in-home and external places (e.g. police department) and external access and/or control by third parties like energy suppliers or emergency staff.

Table 1. Home Automation scenarios

\begin{tabular}{|l|}
\hline Telemetric energy control \\
\hline Telemetric security \\
\hline Telemetric utility meters \\
\hline Baby-care \\
\hline Help to aged persons \\
\hline Automatic disaster reaction \\
\hline External heating/air conditioning control \\
\hline External access to real-time video data \\
\hline Self surveillance \\
\hline Utility contact \\
\hline Security \\
\hline Web cam images for security via E-mail (WISE) \\
\hline $\begin{array}{l}\text { Remote access to kitchen appliances and storage } \\
\text { facilities }\end{array}$ \\
\hline
\end{tabular}




\begin{tabular}{|l|}
\hline Collection and use of environmental data \\
\hline Mood setting \\
\hline Services for disabled people \\
\hline Home networking in a rented home \\
\hline $\begin{array}{l}\text { Multimedia control network with TV interface for } \\
\text { domotic services }\end{array}$ \\
\hline
\end{tabular}

\section{Multimedia / entertainment}

It includes control and exchange of medium to high volume data like video, animation or high-quality audio, in-home and/or with external locations.

Table 2. Multimedia/Entertainment scenarios

\begin{tabular}{|l|}
\hline The wireless WebPAD \\
\hline $\begin{array}{l}\text { VCR programming using TV guide accessed via } \\
\text { Web }\end{array}$ \\
\hline External recording control of video camera data \\
\hline Self surveillance \\
\hline Internet games \\
\hline CD sharing \\
\hline E-mail video orders (EVO) \\
\hline $\begin{array}{l}\text { Multimedia control network with TV interface for } \\
\text { domotic services }\end{array}$ \\
\hline
\end{tabular}

\section{Communication}

It especially covers speech communication (including man-machine interaction) and low volume data exchange.

Table 3. Communication scenarios

\begin{tabular}{|l|}
\hline The wireless WebPAD \\
\hline Baby-care \\
\hline Help to aged people \\
\hline Utility contact \\
\hline Smart answering machine (SAM) \\
\hline Home networking in a rented home \\
\hline
\end{tabular}

\section{Man-machine interface experiences}

The man-machine interfaces have been identified as the major factor for end user acceptance. They have to be easy to use, shall quickly be operational after start-up and have to provide convincing confirmations (live pictures instead of test messages).

Java applets can provide excellent functionality, but this has to be carefully balanced against the corresponding downloading time. Java applications may be the better solution for frequent access to invariable functions from a given location. 
Voice interfaces are very promising, but commercially available speech recognition software is still far from being accurate and easy to use for everybody, especially when e. g. GSM voice quality is not good enough.

$\underline{\text { SMS }}$, remote controls and TV screens have reasonable areas of application, but they are less suited for complex interaction.

WAP is expected to provide a useful addition to a full Web interaction. In the demonstrator, it has successfully been used for environmental status data presentation and remote home appliances control.

Specifically designed hardware and/or software solutions for disabled people can enable these persons to highly benefit from home networking. These solutions have to go far beyond the adaptation of typical I/O devices as demonstrated by mouse operation controlled by a head movement detection system.

\section{Reliability}

Hardware and software reliability is an important issue especially in the typically nonexpert home environment. Most of the commercial equipment used to implement the demonstrator sufficiently fulfilled this requirement.

Microsoft Windows 95/98 during our tests showed not to be stable enough for a home server, Linux or Windows NT are better suited.

Concurrent applications may be a problem for the computers typically used in home environments, distribution of these applications onto several hosts may be necessary to reach the required stability (at a higher cost, though).

Powerline transmission may be a good solution for home networking as it does not require additional cabling, but less expensive variants like X10 do not fulfil the reliability requirements of critical applications, e. g. alarm handling. Powerline transmission for data (e.g. PC to PC) is currently not commercially available and should take into account EMC compatibility and regulatory issues.

\section{Security}

Security against unauthorized access to their home is a point where people are generally fairly sensitive about. Nevertheless, typical firewall concepts currently hinder intended access too much to be applicable.

The security service provider concept introduced for the distributed demonstrator seems much more appropriate and could be enhanced by e.g. virus protection mechanisms. More complex access control mechanisms based on origin of access or time of day also easily be integrated.

\section{API implementations}

Existing home applications are based on a number of different APIs that are often proprietary, incompatible and normally just address subsets of the devices that exist in a home. 
Due to the non-availability of API approaches like HomeAPI or OSGi at demonstrator implementation time, CORBA and Java have been used for individual solutions by several HINE partners.

In general, CORBA is suitable for the demonstrator goals, but some mechanisms do not comply with firewalls. Java applets have to be carefully designed to meet man -machine interface requirements (see above).

All partners have used IP as a low level basis. This should be taken into account by unification approaches for the vast number of different low level home networking protocols used today.

\section{Access network basic requirements}

Access network requirements highly depend on the applications used in a given home. Assuming that all scenario categories and information media (text, pictures, video sequences, sound...) will more and more be used in home networking environments, xDSL availability and always-on support will be a growing requirement of residential customers.

xDSL is the only access networking technology provided today for residential customers which can sufficiently adapt to the highly varying bandwidth requirements of home networking applications.

Always-on support is necessary for sufficiently fast and easy external access to home locations.

\section{Overall conclusions}

Besides the specific technical problems described in the sections above, it has generally been concluded that comfortable home networking which covers all relevant areas is possible with today's technologies, but can be still too complex to install and too expensive with reference to certain advanced scenarios. The integration into a single hardware platform of advanced functionalities should be weighted with respected to the scenario to be implemented. It is probably unrealistic the possibility of having flexibility, computing power, low cost, future proofness in a single box, but it is likely to be able in the short term to have a home networking equipment capable of integrating multimedia and data streams (e.g. with IEEE1394 and Ethernet interfaces with the use of IP for everything) and of hosting new bundles of services.

\section{References}

1. http://www.eurescom.de/public/projects/P900-series/P915/P915.htm

2. EURESCOM Project P915-PF Deliverable 1, "Analysis of In-house System Technologies", http://www.eurescom.de/public/projectresults/p900-series/915d1.htm 
3. EURESCOM Project P915-PF Deliverable 2, "Getting the in-house network hooked up to the outside world - constraints, approaches, impacts",

http://www.eurescom.de/public/projectresults/p900-series/915d2.htm

4. EURESCOM Project P915-PF Deliverable 4, "Description and Evaluation of the HINE

Demonstrator", http://www.eurescom.de/public/projectresults/p900-series/915d4.htm 